\title{
Jogo para apoio ao ensino-aprendizagem de especificação de requisitos
}

\author{
João Vitor Demaria Venâncio \\ joaovitorvenancio99@gmail.com \\ Universidade Federal de Santa Catarina \\ Florianópolis, Brazil
}

\author{
Fabiane Barreto Vavassori Benitti \\ fabiane.benitti@ufsc.br \\ Universidade Federal de Santa Catarina \\ Florianópolis, Brazil
}

\begin{abstract}
Requirements Engineering is concerned with identifying, analyzing, documenting and managing software requirements, which is an important phase in the software development process. Research shows that most software design failures are due to requirements engineering issues. Thus, we propose a solution for the active learning of requirements specification techniques. Considering that the user story technique is currently well accepted by IT companies, this paper proposes a mobile game that supports learning and practice in writing user stories.
\end{abstract}

\section{KEYWORDS}

Software Engineering, Requirement Engineering, User Story, Game

\section{INTRODUÇÃO}

O mercado reivindica instituições de ensino para formar futuros colaboradores na área de tecnologia com qualidade [1]. Especialmente nos cursos de graduação, os tópicos de Engenharia de Software são normalmente ensinados de forma bastante superficial, envolvendo poucas disciplinas no curso [2] e com pouco alinhamento com as necessidades da indústria [3].

Estatísticas demonstram que mais de $50 \%$ dos casos de falha em projetos de software são causadas por uma gerência de requisitos imatura ou inadequada [4]. Isto ocorre pois o processo de Engenharia de Requisitos é repleto de dificuldades, principalmente nas etapas de elicitação e especificação dos requisitos do software. $\mathrm{O}$ não entendimento das expectativas dos clientes e, consequentemente, a especificação incoerente de requisitos são fatores críticos para o insucesso do projeto de software [5].

Neste contexto, destaca-se a necessidade de desenvolver soluções para apoiar o processo de ensino-aprendizagem da Engenharia de Requisitos. O estado da arte referente ao ensino em engenharia de requisitos, bem como os conceitos básicos a serem explorados pela solução proposta estão descritos na seção 2. A seção 3 apresenta a descrição da solução proposta e, por fim, na seção 4 as considerações finais.

\section{BACKGROUND}

\subsection{Estado da arte}

A partir dos resultados de um mapeamento sistemático cujo objetivo foi apresentar as pesquisas atuais sobre Educação em Engenharia de Requisitos disponíveis e selecionar abordagens para futuras pesquisas [6], observa-se na Figura 1 as abordagens de ensino da Engenharia de Requisitos. Pode-se constatar que apenas uma ferramenta foi proposta como solução para a especificação de requisitos. A ferramenta em questão se refere a uma simulação em planilha eletrônica de um microprocessador (computador) no nível de requisitos [7].

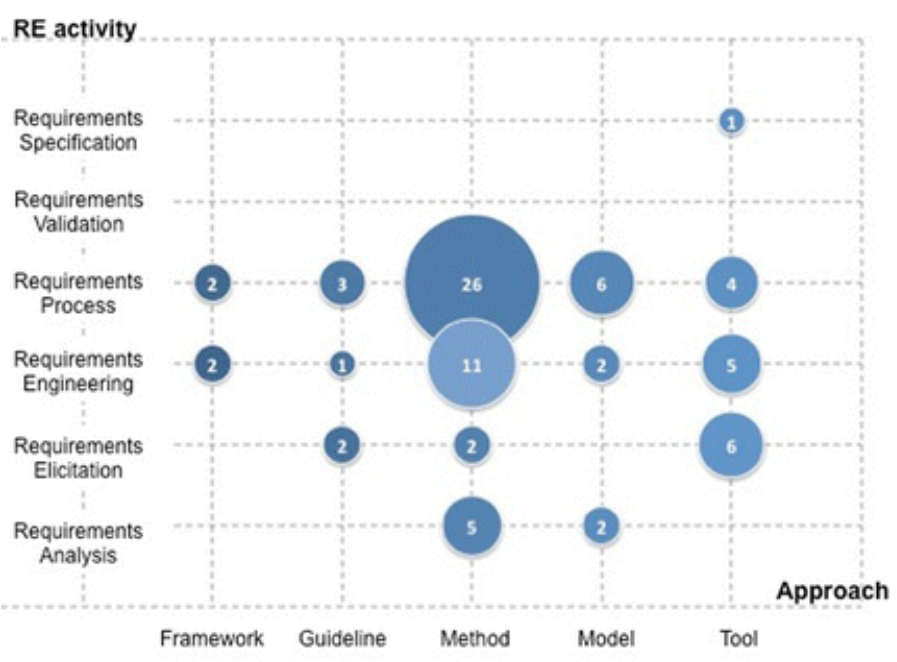

Figura 1: A Pesquisa em Educação em Engenharia de Requisitos

Estudo semelhante foi realizado com foco apenas nas soluções nacionais, visando identificar as soluções para apoio ao ensino de Engenharia de Software [8]. Os autores identificaram 14 soluções com foco na área de Engenharia de Requisitos, sendo que apenas uma delas [9] se concentra na Especificação dos Requisitos, especificamente utilizando diagramas da UML.

Uma pesquisa com 25 empresas de TI do Brasil [10] apontou que a história de usuário (user story) está entre as técnicas mais empregadas para especificação de requisitos, tendo sido reportado o uso por $52 \%$ das empresas. Observa-se, a partir das pesquisas listadas acima, que esta técnica bastante utilizada não é contemplada por nenhuma das soluções educacionais encontradas. Desta forma, a seção seguinte apresenta os conceitos básicos desta técnica e, na sequencia, a proposta de uma solução de apoio para o ensino/aprendizagem da técnica de especificação por histórias de usuário é apresentada.

\subsection{User Story}

Uma história de usuário consiste em um texto simples e curto que descreve uma funcionalidade do sistema computacional a partir da perspectiva de seus usuários. A estrutura dele pode ser descrita da seguinte forma: no início é identificado o sujeito para quem o sistema irá realizar alguma tarefa; depois é descrita funcionalidade 
desejada; por fim é exposto qual o benefício que essa funcionalidade vai proporcionar ao usuário, sendo esta última parte opcional [11]. Dessa forma, tem-se o seguinte padrão para uma história do usuário:

Como um [usuário do sistema],
eu gostaria de [realizar esta função]
(opcional: para que eu possa [justificativa do
benefício]).

\section{SOLUÇÃO PROPOSTA}

Para auxiliar no processo de ensino-aprendizagem da Engenharia de Requisitos é proposto a criação de um jogo digital para dispositivos móveis que auxilie no aprendizado de elicitação e escrita de histórias de usuário. Nele o estudante (jogador) é inserido em cenários onde ele deverá propor soluções em forma de histórias de usuários para problemas e dificuldades de stakeholders, pessoas que procuram a criação de um software que o ajude na sua profissão ou no seu dia-a-dia. Dessa forma, o estudante é colocado em situações às quais ele poderá exercer a especificação de requisitos para um cliente, auxiliando na sua formação acadêmica (Figura 2(a)).

Para criar um ambiente competitivo e instigar o usuário a dar o seu melhor, é implementado um ambiente multijogador no qual os jogadores vão estar competindo pelo "louvor"do stakeholder, definindo entre eles quem melhor entendeu a necessidade do stakeholder e redigiu as melhores histórias de usuário. Portanto, o jogador deverá ficar atento as necessidades do seu contratante, a fim de elaborar a melhor história de usuário possível entre os seus oponentes.

Uma partida do jogo funciona por turnos, sendo cada turno composto por duas fases: na primeira fase o jogador procura entender os problemas do stakeholder (Figura 2(b)), propondo uma história de usuário (Figura 2(c)). Na segunda fase o jogador avalia as histórias criadas pelos demais jogadores, precisando escolher a melhor dentre elas (Figura 2(d)). No final de cada turno, os jogadores que tiveram as melhores histórias avaliadas serão aqueles que receberão a maior pontuação. Cada novo turno é um novo desafio proposto pelo stakeholder. Com isso, no final da partida, quando se passarem todos os turnos, ter-se-á um ranking com os jogadores que souberam escrever as melhores histórias, sendo o primeiro lugar o ganhador de um contrato de exclusividade de desenvolvimento software com o stakeholder.

Caso algum dos jogadores não estiver se saindo bem no jogo, o Mike - personagem inspirado no Mike Cohn [11], que ajudou na criação do conceito e na propagação do uso das histórias de usuário, vai aparecer para ajudar o jogador apresentando dicas de como melhorar a qualidade das suas histórias.

Para o desenvolvimento está sendo utilizado o motor de jogo Unity e a base de dados Firebase da Google para implementar as funcionalidades do multijogador. A Unity foi escolhida como ferramenta porque, além de possuir licenças gratuitas para estabelecimentos de ensino, ela oferece a compilação de um mesmo projeto de jogos para múltiplas plataformas, como o iOS e o Android [12]. Pela característica do jogo funcionar em turnos e a facilidade que a ferramenta oferece em poder alterar ou adicionar novos stakeholders e desafios diretamente na base de dados, o Firebase foi escolhido [13].

\section{CONSIDERAÇÕES FINAIS}

Com a criação do jogo e no uso dele como ferramenta para auxiliar no processo de ensino-aprendizagem de Engenharia de Requisitos, espera-se que os alunos tenham um melhor aproveitamento dos conteúdos ministrados em sala ao inseri-los em um contexto onde se possa competir e aplicar o conteúdo sobre histórias de usuário, formando profissionais mais capacitados os quais ajudariam num aumento da taxa de sucesso dos projetos de software.

Atualmente o projeto se encontra em etapa de implementação, no desenvolvimento do sistema de gerenciamento de partidas do ambiente multijogador, para permitir que as pessoas consigam se conectar em uma sala e iniciar um jogo.

\section{ACKNOWLEDGMENTS}

O presente trabalho foi realizado com o apoio do Conselho Nacional de Desenvolvimento Científico e Tecnológico - CNPq - Brasil

\section{REFERÊNCIAS}

[1] M. da C. O. de Souza, S. R. B. Oliveira, and S. R. L. Meira. A systematic review to assist in identifying teaching approaches to guide the application of an interdisciplinary software factory in it undergraduation. In Proceedings of the 31st Brazilian Symposium on Software Engineering, SBES'17, pages 384-391, New York, NY, USA, 2017. ACM. ISBN 978-1-4503-5326-7. doi: 10.1145/3131151.3131176. URL http://doi.acm.org/10.1145/3131151.3131176.

[2] C.G. Von Wangenheim and D.A Silva. Qual conhecimento de engenharia de software é importante para um profissional de software? In Anais Fórum de Educação em Engenharia de Software, 2009.

[3] E. Tuzun, H. Erdogmus, and I.G. Ozbilgin. Are computer science and engineering graduates ready for the software industry?: Experiences from an industrial student training program. In Proceedings of the 40th International Conference on Software Engineering: Software Engineering Education and Training, ICSE-SEET '18, pages 68-77, New York, NY, USA, 2018. ACM. ISBN 978-1-4503-5660-2. doi: 10.1145/3183377.3185754. URL http://doi.acm.org/10.1145/3183377.3185754.

[4] A. Van Lamsweerde. Requirements Engineering: From System Goals to UML Models to Software Specifications. Wiley, 2009.

[5] The Standish Group. The standish group report-chaos, 2015.

[6] S. Ouhbi, A. Idri, J.L. Fernández-Alemán, and A. Toval. Requirements engineering education: a systematic mapping study. Requirements Engineering, 20(2):119138, Jun 2015. ISSN 1432-010X. doi: 10.1007/s00766-013-0192-5. URL https: //doi.org/10.1007/s00766-013-0192-5

[7] H.T. Salzer and I. Levin. Spreadsheet-based logic controller for teaching fundamentals of requirements engineering. International fournal of Engineering, 20(6), 2004.

[8] J.A.O.G. da Cunha, G.A. Marques, W.L. Lemos, U.D. Câmara, Jr, and F.J.S. Vasconcellos. Software engineering education in brazil: A mapping study. In Proceedings of the XXXII Brazilian Symposium on Software Engineering, SBES '18, pages 348-356, New York, NY, USA, 2018. ACM. ISBN 978-1-4503-6503-1. doi: 10.1145/3266237.3266259. URL http://doi.acm.org/10.1145/3266237.3266259.

[9] J.C. Silva, S.P.A. Sousa, R. Kulesza, and A.V. Brito. Uma avaliação do emprego do jogo modelando como apoio ao ensino de engenharia de requisitos. In Anais do XX Workshop sobre Educação em Informática, 2012.

[10] F.B.V. Benitti. As a teacher, i want to know what to teach in requirements engineering so that professionals can be better prepared. In Proceedings of the 31st Brazilian Symposium on Software Engineering, SBES'17, pages 318-327, New York, NY, USA, 2017. ACM. ISBN 978-1-4503-5326-7. doi: 10.1145/3131151.3131185. URL http://doi.acm.org/10.1145/3131151.3131185.

[11] M. Cohn. Mountain Goat Software: User Stories, 2019. URL https://www. mountaingoatsoftware.com/agile/user-stories. Acesso em: 2019-10-17.

[12] Unity Technologies. Unity Real-Time Development Platform | 3d, 2d vr ar visualizations, 2019. URL https://unity.com/. Acesso em: 2019-10-16.

[13] Google. Firebase, 2019. URL https://firebase.google.com/?hl=pt-br. Acesso em: 2019-10-16. 


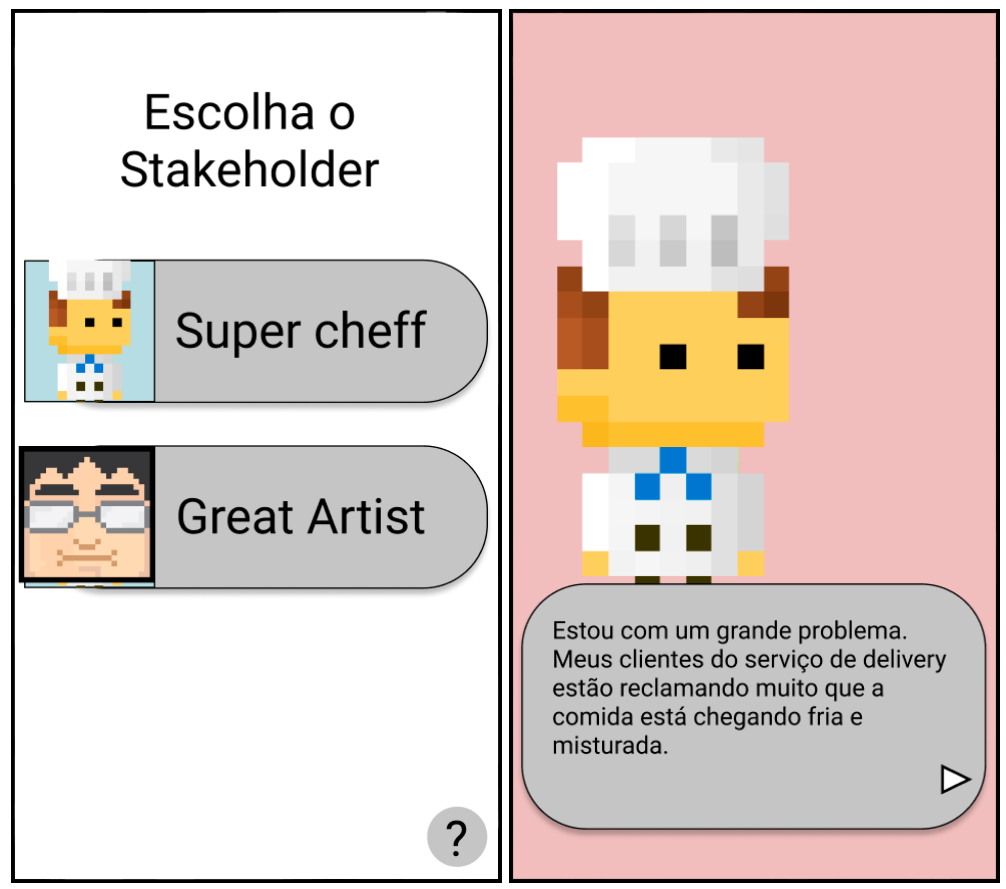

(a) Escolha do desafio

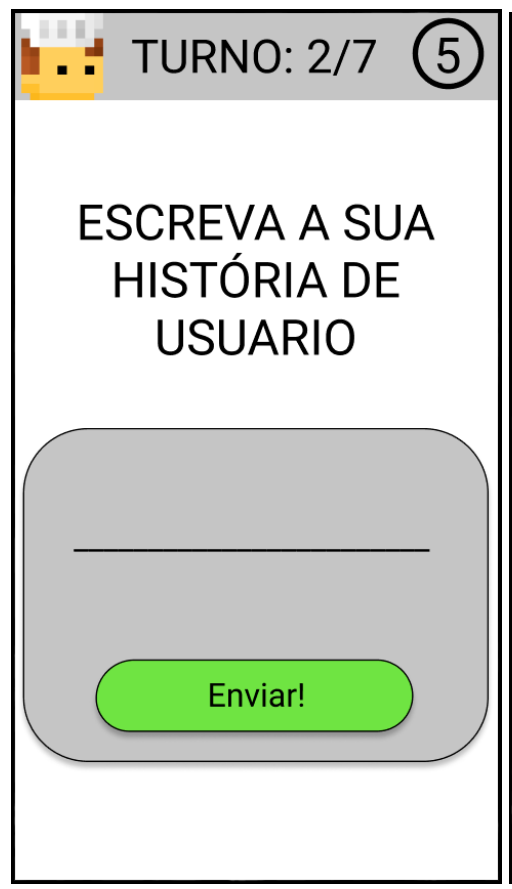

(c) Escrevendo história (b) Necessidade do stakeholder

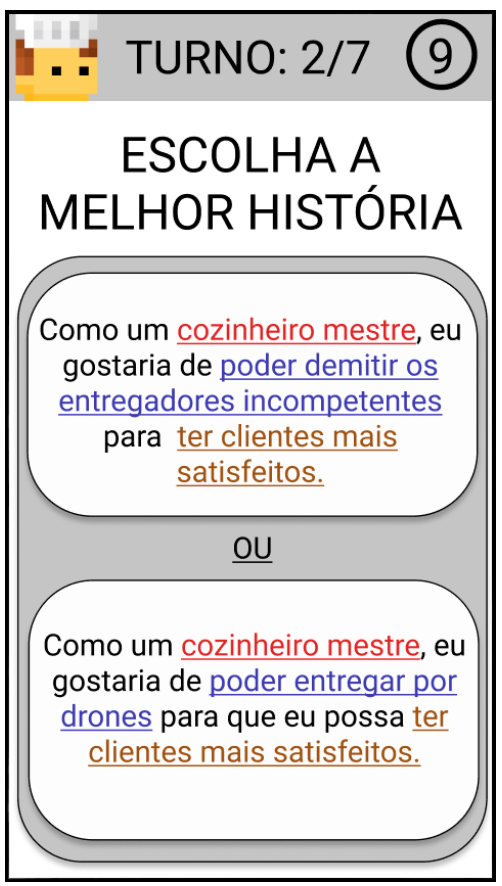

(d) Avaliando histórias

Figura 2: Protótipos da solução proposta 